\title{
CONTROL TÁCTIL ADAPTABLE PARA LA MANIPULACIÓN DE OBJETOS DEFORMABLES
}

\author{
Ángel Delgado \\ Instituto Universitario de Investigación Informática \\ Universidad de Alicante \\ angel.delgado@ua.es \\ Carlos A. Jara, Fernando Torres \\ Departamento de Física, Ingeniería de Sistemas y Teoría de la Señal \\ Universidad de Alicante \\ \{carlos.jara, fernando.torres\}@ua.es
}

\begin{abstract}
Resumen
El agarre y la manipulación de objetos deformables con manos robóticas dependen de las características fisicas del objeto tratado. Estas características físicas son frecuentemente desconocidas, por lo que es necesaria la utilización de un sistema de control adaptable, que permita estabilizar el agarre y realizar tareas de manipulación controlando las fuerzas ejercidas. Las posiciones de los dedos y las fuerzas aplicadas se deben adaptar a la deformación causada o deseada. En este artículo se presenta un sistema de control basado en información táctil, en el que el movimiento de cada dedo de una mano se controla para mantener un valor de fuerza. Este valor de fuerza depende de las características físicas del objeto, en concreto su dureza y grado de deformación
\end{abstract}

Palabras Clave: Manipulación en mano, agarre, control táctil, objetos deformables.

\section{INTRODUCCIÓN}

En el campo de la manipulación robótica, el estudio de cómo agarrar y manipular objetos deformables es un reto abierto $\mathrm{y}$ ha sido estudiado en diferentes contextos, desde la industria a la cirugía [7]. Hoy en día, la capacidad de un sistema robótico para manipular objetos deformables es una importante característica buscada para conseguir robots más versátiles en distintos ambientes de trabajo.

Mientras que para la manipulación de objetos rígidos los movimientos generados por los dedos de la mano se pueden predecir con los principios de movimiento para el objeto rígido, teniendo en cuenta las posiciones de los contactos y las fuerzas ejercidas [18], para un objeto deformable, estos principios no son aplicables. La utilización de la matriz de agarre y las matrices jacobianas de los dedos de la mano no son suficientes, en el caso de la manipulación de objetos deformables, para modelar tanto los movimientos como las deformaciones causadas. Por lo tanto el sistema manoobjeto no puede ser controlado usando las mismas leyes que las que se usan con objetos rígidos. El comportamiento dinámico de los objetos deformables depende de un número amplio y complejo de parámetros tales como la dureza, fricción o elasticidad.

Para la mayoría de los objetos deformables es posible obtener un modelo exacto usando alguna de las técnicas habituales como pueden ser los modelos masa-muelle [3], modelos mesh-less [4] o el método basado en elementos finitos (FEM) [6]. A pesar de que son técnicas precisas, la mayor parte necesitan una resolución exacta del modelo, lo cual necesita tiempos de procesamiento largos y por lo tanto puede ser problemático cuando se intentan utilizar estrategias de control en tiempo real. Teniendo en cuenta estas limitaciones, se han desarrollado otros enfoques para la manipulación de objetos deformables en los que se utiliza un modelo simplificado del objeto o, en otros casos, sin recurrir a la utilización de modelado $[5,14$, 15]. En este último caso, la utilización de información táctil para obtener información sobre la dureza y grado de deformación, y como procesar dicha información para usarla de referencia para controlar los movimientos de los dedos, son temas de estudio claves para obtener un sistema de control fiable. Este campo de estudio se inspira en el mecanismo humano para realizar tareas de agarre y manipulación, que esencialmente depende la sensación táctil, más que las otras percepciones utilizadas, visión y propiocepción $[2,8]$.

Generalmente, el agarre y la manipulación de objetos deformables dependen de un sistema multisensorial $[9,12]$, que se utiliza para control el estado del objeto en cada momento del proceso. La propia información (cinemática y dinámica) del dispositivo de manipulación robótica, información visual e 
información táctil se usan conjuntamente para controlar el sistema. En una parte de los trabajos desarrollados hasta el momento, solo se recurre a la información interna del dispositivo manipulador y a la información táctil para controlar la ejecución de las tareas. En estos casos, la fiabilidad del sistema depende de la precisión y funcionamiento del sensor táctil utilizado, para obtener una realimentación fiable de cómo evolucionan las fuerzas en los puntos de contacto $[1,11,14,16]$. Los sensores táctiles proporcionan el valor de referencia de la fuerza, tanto en posición como en magnitud, que pueden usar los controladores de las articulaciones de la mano, para cada uno de los contactos con el objeto [10]. En muchos casos, estos sistemas están basados en algoritmos de aprendizaje sin tener en cuenta las características del objeto, o recurren al uso de modelos complejos del objeto.

En este artículo se presenta una nueva técnica para estabilización del agarre y manipulación en mano de objetos deformables elásticos isotrópicos. Esta técnica se basa en la utilización de información proveniente de un sensor táctil. El control táctil permite tanto prevenir la perdida de contacto en los puntos de agarre, como ajustar la magnitud o posición de la fuerza ejercida en cada punto de contacto con el objeto. Como referencia para el control táctil se utiliza el módulo de Young para los objetos, que relaciona las fuerzas ejercidas en el objeto con la deformación provocada. Este valor es conocido o se estima en una etapa previa de extracción de características del objeto.

\section{DESCRIPCIÓN DEL SISTEMA ROBÓTICO UTILIZADO}

\subsection{MANO Y BRAZO ROBÓTICOS}

Para el desarrollo de los experimentos se ha usado el sistema robótico descrito en la Figura 1. El sistema se compone de un robot para manipulación Mitsubishi PA10 con siete articulaciones, y la mano robótica Shadow [19], con sensores táctiles Tekscan Grip [20]. El sensor táctil se ha añadido a la mano robótica para obtener información de fuerza y presión en los dedos de la mano. La mano Shadow se monta en el extremo del robot articulado, lo que permite obtener un espacio de trabajo amplio para las tareas de manipulación.

La mano robótica Shadow es un dispositivo robótico actuado con motores. El movimiento de los dedos se obtiene con la utilización de transmisiones de cable conectados a los motores y a cada articulación. Con este diseño se obtiene un dispositivo que simula en tamaño y configuración de movimientos a la mano humana. La mano dispone de 20 grados de libertad ( 2 en la muñeca, 5 en el dedo pulgar, 3 en los dedos índice, medio y anular, y 4 en el dedo meñique). Para simular el comportamiento de una mano humana, las últimas dos articulaciones correspondientes a los extremos de los dedos (excepto el pulgar) están acopladas, es decir, las dos articulaciones están comandadas por un único valor de posición. Teniendo en cuenta esto, la mano dispone de 24 grados de libertad, pero solo 20 se controlan directamente. Para cada una de estas articulaciones se usa un control de posición. Se dispone del modelo cinemático de la mano para calcular soluciones cinemáticas directas e inversas.

Además del sistema real, se dispone de modelos 3D del sistema para simular movimientos de la mano y el brazo robótico, usando el simulador Gazebo y la herramienta de planificación de trayectorias MoveIt de ROS. Sin embargo, debido a las deformaciones que se producen en el objeto, este software no permite obtener simulaciones realistas del comportamiento del conjunto mano-objeto, con lo que se ha recurrido a experimentos reales para demostrar la funcionalidad de la técnica de control presentada.

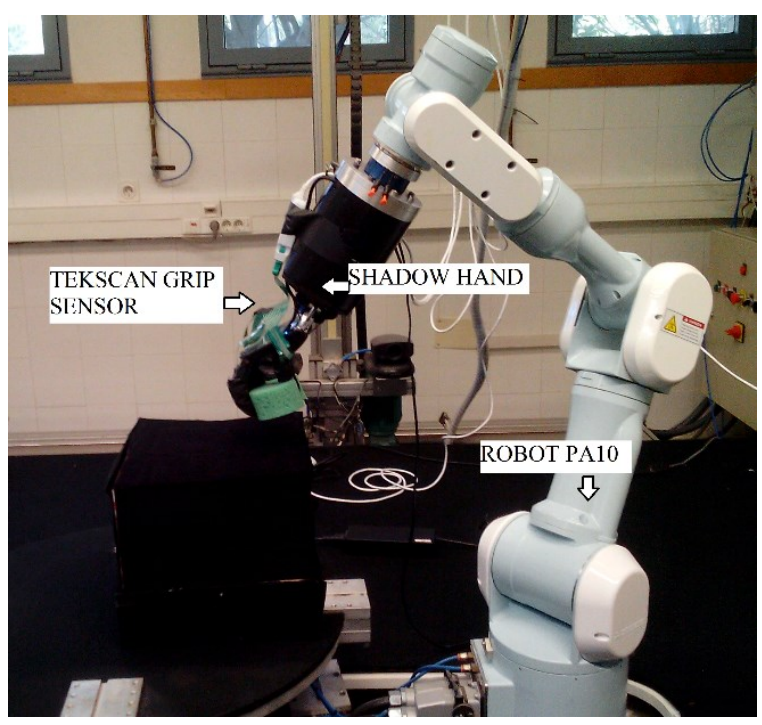

Figura 1: Imagen general del sistema robótico compuesto por la mano Shadow, el robot PA10 y el sensor táctil Tekscan.

\subsection{SENSOR TÁCTIL}

En cuanto al sensor táctil Tekscan Grip, se trata de un sensor con forma adaptable a una mano (humana o robótica) con cinco dedos y la palma. Se usa para obtener el mapa de presión de la mano (Figura 2). El sensor se divide 18 regiones que cubren los dedos y la palma. Cada región se compone de una matriz de elementos de captación (tactels) de distintas dimensiones. En este artículo se consideran solo configuraciones en las que el agarre del objeto se realiza con los extremos de los dedos, por lo que para los controladores táctiles se usan solo las regiones 
correspondientes a los extremos. Estas regiones se componen de una matriz de $4 \times 4$ elementos, y tienen una dimensión de 1'7 x 1'7 centímetros. Los sensores se han calibrado para obtener valores de presión / fuerza reales. Con esta calibración se obtienen curvas de calibración para cada región. La respuesta de las regiones se aproxima a una relación lineal hasta el valor límite de 7 newtons. El sensor se conecta a un ordenador mediante una conexión Ethernet local. La tasa de muestreo está alrededor de los $850 \mathrm{~Hz}$.

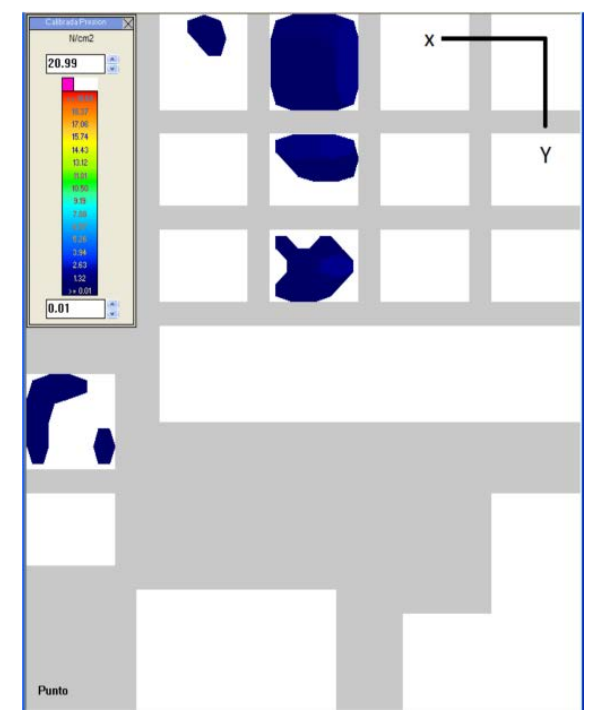

Figura 2: Visualización del mapa de presión de la mano ofrecido por el sensor táctil Tekscan Grip.

\section{CONTROL TÁCTIL ADAPTABLE PARA MANIPULACIÓN DE OBJETOS DEFORMABLES}

En esta sección se presenta la estructura de control definida para usar en tareas de manipulación de objetos deformables. El objetivo de esta técnica es controlar el movimiento de los dedos en función del valor táctil obtenido en cada momento, lo cual permite por un lado estabilizar posiciones de agarre $\mathrm{y}$ reaccionar a deslizamientos del objeto, y por otro lado reajustar las fuerzas ejercidas por cada dedo en función de la deformación buscada.

Para esta última parte, en la que se controla la deformación deseada, se usa como referencia el valor del Módulo de Young para objetos deformables isotrópicos, un módulo de elasticidad obtenido como el coeficiente entre la presión ejercida y la deformación generada. El valor del módulo de Young es un valor que ha de ser obtenido con experimentación directa sobre el objeto. Como este valor es desconocido frecuentemente, se presenta en la siguiente subsección un método de exploración que permite estimar este valor y usarlo en el control táctil.

\subsection{EXTRACCIÓN DE CARACTERÍSTICAS DEL OBJETO}

Como se ha comentado anteriormente, en los casos en los que no se dispone del valor del Módulo de Young para el objeto manipulado, se utiliza una etapa previa de exploración y extracción de características del objeto. Con esta etapa se obtiene una estimación del valor real para dicho objeto.

El proceso de extracción de características empieza con la mano situada en una posición de agarre del objeto. Los puntos de contacto iniciales se seleccionan a partir de medidas de calidad del agarre, mediante un procedimiento cuya descripción queda fuera del ámbito de este artículo [13]. El proceso se basa en la ejecución de un algoritmo de movimiento secuencial de los dedos, limitado por el máximo desplazamiento posible para cada uno de ellos, o la fuerza máxima que se puede aplicar. Experimentalmente se establece esta fuerza en 3 newtons, que es la fuerza táctil obtenida cuando se aplica el máximo par a las articulaciones de los dedos presionando un objeto rígido. El proceso de extracción de características se basa en el comportamiento humano y la ley de Hooke para objetos elásticos, que relaciona fuerzas ejercidas don deformaciones provocadas. El ser humano realiza la misma acción para estimar cuánto se puede deformar un objeto, es decir, presionarlo y relacionar la fuerza que se está sintiendo en los dedos, y cuanto se han desplazado éstos. El algoritmo de exploración del objeto genera movimientos iterativos para cada uno de los dedos en contacto con el objeto. Posteriormente, se obtiene la estimación del módulo de Young, que define la elasticidad del objeto, como la relación entre la presión final ejercida leída en los sensores $\sigma_{i}$, y la deformación generada. Ya que el contacto entre dedo y objeto se ha mantenido, por la presencia de un valor de fuerza continuado, el valor de la deformación $\epsilon_{i}$ causada se hace corresponder con el desplazamiento del extremo de cada dedo, obtenido con información cinemática interna de la mano. Para cada uno de los dedos utilizados se obtiene un valor de la aproximación del módulo de Young $K^{\sim}{ }_{d_{i}}$. En caso de pérdida de contacto, el proceso de estimación sería inválido.

$$
K^{\sim} d_{i}=\frac{\sigma_{i}}{\epsilon_{i}}
$$

Para obtener una estimación más robusta del valor de deformación, una vez obtenido el valor para cada dedo, se obtiene el valor óptimo como la media de los valores para cada dedo. Con esto se pretende equilibrar los posibles errores producidos por respuestas distintas de los sensores en las distintas regiones. 


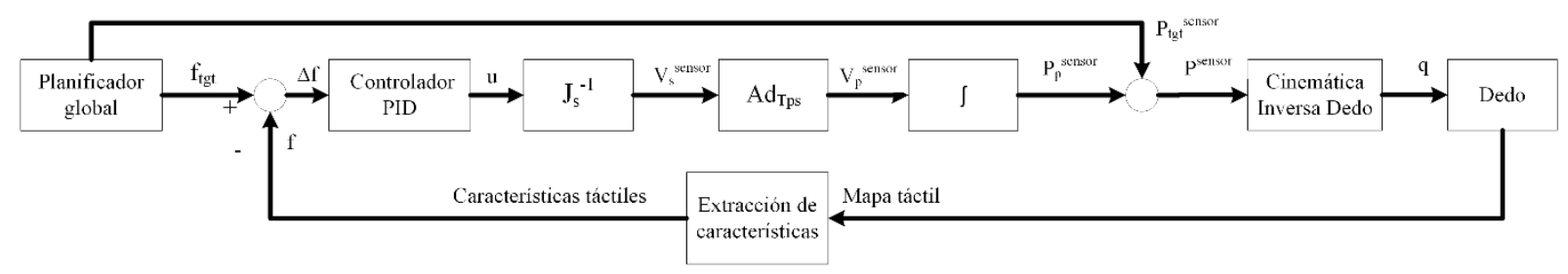

Figura 3: Diagrama del control servo-táctil para cada dedo.

\subsection{CONTROL SERVO-TÁCTIL PARA MANIPULACIÓN DIESTRA DE OBJETOS DEFORMABLES}

Para tareas de manipulación diestra que impliquen únicamente movimientos de los dedos, es necesario definir las posiciones y fuerzas ejercidas por los dedos en cada iteración. Cada tarea puede requerir añadir o eliminar contactos con el objeto, cambiar la posición del contacto o cambiar la fuerza aplicada en alguno de los contactos.

Para controlar cada uno de los dedos, se utiliza un controlador servo-táctil. Las características que se desea controlar son la posición y magnitud de la fuerza ejercida en el contacto. La magnitud se obtiene directamente desde el sensor táctil, y la posición se obtiene como la posición de la celda del sensor de cada región del sensor con el valor máximo. Para obtener una deformación deseada en un objeto deformable, se calcula la fuerza necesaria a ejercer teniendo en cuenta el valor del módulo de Young del objeto, valor que se conoce o se ha aproximado según el proceso descrito en la Sección 3.1. La Figura 3 describe el controlador servo-táctil.

La misión del controlador es obtener la velocidad del sensor $\boldsymbol{V}_{\boldsymbol{s}}^{\boldsymbol{s e n} \boldsymbol{s}} \in \mathbb{R}^{\mathbf{3}}$ que minimiza el error en las características deseadas $\boldsymbol{\Delta f} \in \mathbb{R}^{\mathbf{3}}$. En el caso de los dedos robóticos de una mano, las articulaciones no permiten movimientos de rotación significativos, por lo que solo se controla la velocidad lineal. La salida del controlador, dada como velocidad del extremo, se integra según el tiempo de ciclo para obtener la nueva posición de la articulación para obtener el contacto deseado. Usando la cinemática de los dedos, las nuevas posiciones de las articulaciones se controlan con los controladores de posición de la mano Shadow.

El vector de características $\Delta \mathrm{f}(\mathrm{t})=\left[\boldsymbol{\Delta} \boldsymbol{f}_{\boldsymbol{x}}, \boldsymbol{\Delta} \boldsymbol{y}, \boldsymbol{\Delta} \boldsymbol{f _ { m }}\right] \in$ $\mathbb{R}^{3}$ incluye los valores de error respecto a las características deseadas $\mathbf{f}_{\text {tgt }}=\left[\boldsymbol{f} \_\boldsymbol{x}_{\boldsymbol{t} t \boldsymbol{t}}, \boldsymbol{f} \_\boldsymbol{y}_{\boldsymbol{t} t \boldsymbol{t}}, \boldsymbol{f} \_\boldsymbol{m}_{\text {tgt }} \boldsymbol{t} \in\right.$ $\mathbb{R}^{\mathbf{3}}$. Las características de posición $\left(\boldsymbol{f}_{\boldsymbol{x}}, \boldsymbol{f}_{\boldsymbol{y}}\right)$ se establecen como las coordenadas de la celda de la región correspondiente del sensor con el valor máximo de fuerza, y la magnitud se establece como la fuerza resultante en el área del sensor $\boldsymbol{A}_{\text {sensor, }}$ es decir la suma de los valores de todas las celdas.

El vector de error $\Delta \mathbf{f}(\mathbf{t})=\left[\boldsymbol{\Delta} \boldsymbol{f}_{\boldsymbol{x}}, \boldsymbol{\Delta} \boldsymbol{f} \boldsymbol{y}, \boldsymbol{\Delta} \boldsymbol{f}_{\boldsymbol{m}}\right] \in \mathbb{R}^{\mathbf{3}}$ incluye los valores del error respecto a los valores deseados $\mathbf{f}_{\text {tgt }}$ $=\left[\boldsymbol{f} \_\boldsymbol{x}_{\boldsymbol{t} t \boldsymbol{t}}, \boldsymbol{f} \_\boldsymbol{y}_{\boldsymbol{t} \boldsymbol{t} t,} \boldsymbol{f} \_\boldsymbol{m}_{\text {tgt }}\right] \in \mathbb{R}^{\mathbf{3}}$. El valor deseado en posición $\left(\boldsymbol{f} \_\boldsymbol{x}_{t g t}, \boldsymbol{f}_{-} \boldsymbol{y}_{t g t}\right)$ se establece como la posición de la celda central en la región del sensor correspondiente a la yema de los dedos, con el fin de mantener centrado el contacto en el dedo. El valor deseado de magnitud $\left(\boldsymbol{f}_{-} \boldsymbol{m}_{\text {tgt }}\right)$ se establece adaptativamente según el valor deseado de deformación en el objeto, es decir, este valor se establece de acuerdo con el valor del módulo de Young para el objeto manipulado.

La base de cada controlador para cada dedo es un controlador PID que minimiza el error respecto a las características deseadas $\boldsymbol{\Delta}$ f. Cada una de las características se controla con una configuración distinta para los valores $\mathrm{p}$, i y d. Los valores de estos parámetros para controlar el error en la magnitud de la fuerza $\boldsymbol{\Delta} \boldsymbol{f}_{\boldsymbol{m}}$ se establecen para obtener una respuesta suave, que evite deformaciones bruscas y no deseadas en el objeto, ya que las oscilaciones en el movimiento de los dedos provocan este tipo de comportamiento. La variable de control $\boldsymbol{u}$ proporcionada por el controlador, se usa en la siguiente etapa para obtener el movimiento de los dedos.

Para traducir la respuesta del controlador a una velocidad respecto al sensor que minimice el error, se define una matriz de interacción $\boldsymbol{J}_{\boldsymbol{S}}^{-\mathbf{1}} \in \mathbb{R}^{\mathbf{3 x} \mathbf{3}}$. Los ejes en el plano del sensor $(x, y)$ se corresponden respectivamente con los ejes $X_{s} \mathbf{y} Z_{s}$ del sensor $O_{s}$. Usando esta configuración, los errores en la posición de la fuerza se corresponden con los ejes $X_{s}$ y $Z_{s}$. En cuanto a la correspondencia de la magnitud del error, se han probado distintas configuraciones de la matriz de interacción $\boldsymbol{J}_{\boldsymbol{s}}^{\mathbf{1}}$ para obtener un movimiento apropiado y realista en los dedos. Se ha comprobado que si el error en magnitud se mapea solo al eje $Z_{s}$ de $O_{s}$ los movimientos del dedo no permiten obtener el comportamiento deseado. El error debe ser mapeado con los ejes $Z_{s}$ e $Y_{s}$, para obtener una trayectoria de apertura o cierre del dedo que pueda ser seguida por el dedo robótico. En el caso del dedo pulgar, los valores de la matriz de interacción son negativos, ya que el pulgar se mueve en el sentido 
opuesto al resto de los dedos, para producir un movimiento de cierre o apertura en la mano. La siguiente ecuación muestra cómo se mapea el error a la velocidad del sensor.

$$
\begin{gathered}
V_{s}^{\text {sens }}=J_{s}^{-1} \cdot \Delta f \\
=\left(\begin{array}{lll}
1 & 0 & 0 \\
0 & 1 & 0 \\
0 & 1 & 1
\end{array}\right) \cdot\left(\begin{array}{l}
\Delta f_{x} \\
\Delta f_{m} \\
\Delta f_{y}
\end{array}\right)
\end{gathered}
$$

Considerando el controlador PID, la velocidad obtenida $\boldsymbol{V}_{\boldsymbol{S}}^{\boldsymbol{s e n s}}=\left[\boldsymbol{V}_{\boldsymbol{x}}, \boldsymbol{V}_{\boldsymbol{y}}, \boldsymbol{V}_{z}\right] \in \mathbb{R}^{\mathbf{3}}$ es:

$$
\begin{gathered}
V_{s}^{\text {sens }}=J_{s}^{-1} . \\
{\left[K_{p} \cdot \Delta f(t)+\cdot K_{i} \int \Delta f(t) d t+\right.} \\
\left.\cdot K_{d}(\Delta f(t)-\Delta f(\mathrm{t}-1))\right]
\end{gathered}
$$

La velocidad obtenida respecto al eje de coordenadas del sensor $O_{s}$ se transforma en a la velocidad $\boldsymbol{V}_{s}^{\text {palm }} \in$ $\mathbb{R}^{\mathbf{3}}$ en el sistema de referencia para cada dedo, situado en la palma de la mano robótica, $O_{p}$. Para realizar la transformación en velocidad se utiliza la matriz

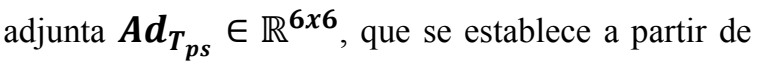
las matrices de transformación entre $O_{p}$ y $O_{s}$ :

$$
A d_{T_{p s}}=\left(\begin{array}{cc}
R_{p s} & \hat{p}_{p s} R_{p s} \\
0 & R_{p s}
\end{array}\right)
$$

Usando la velocidad del sensor respecto al sistema de la palma de la mano, la nueva posición para cada extremo de cada dedo $\left(\boldsymbol{P}_{s}^{\text {palm }} \in \mathbb{R}^{\mathbf{3}}\right.$ ) se obtiene integrando el valor de cada eje entre el instante de tiempo $t$ y $t-1$, y la posición para cada articulación (q) se obtiene con el uso de la cinemática inversa de la mano.

A nivel alto, se establece la posición deseada y la fuerza a ejercer por cada dedo en cada etapa de una tarea de manipulación. Cada etapa de una tarea de manipulación se define con una configuración en los dedos y en las fuerzas a ejercer. En este artículo solo se discute cómo esta planificación cambia las fuerzas deseadas en cada dedo. El control de la deformación en el objeto se basa en la ley de Hooke para objetos elásticos, usando el valor real $K$ o el valor aproximado $K^{\sim}$ que describe el objeto:

$$
\begin{gathered}
\sigma_{d}=K^{\sim} * \epsilon_{d} \\
F_{d}=\sigma_{d} * A_{\text {sensor }}
\end{gathered}
$$

esa deformación. Este valor de presión se traduce en el valor de fuerza deseada $\boldsymbol{F}_{\boldsymbol{d}}$ para el controlador servo-táctil de cada dedo, teniendo en cuenta el área del sensor $\boldsymbol{A}_{\text {sensor }}$.

\section{EXPERIMENTACIÓN}

En esta sección se describen algunas de las pruebas realizadas para testear el algoritmo de reconocimiento descrito en la Sección 3.1 y el control servo-táctil descrito en la Sección 3.2. Se han realizado pruebas con distintos tipos de objetos deformables elásticos, con valores de densidad y elasticidad desconocidos. En los experimentos se asume que los dedos se sitúan en una posición inicial de agarre, y que la configuración inicial es válida para agarrar el objeto. En la primera subsección se incluyen experimentos para la primera etapa de exploración del objeto. En la segunda subsección se documentan experimentos para testear el control servo-táctil.

\subsection{EXPERIMENTOS PARA LA EXTRACCIÓN DE CARACTERÍSTICAS DE LOS OBJETOS}

En esta sección se muestran resultados para la parte de extracción de características usando distintos tipos de objetos, con distintas formas y densidades. Para todos los experimentos los valores de fuerza y desplazamiento del dedo pulgar (F5) difieren en gran medida de los valores para el resto de dedos (F1, F2, F3, F4). Esto es debido a la funcionalidad distintiva del dedo pulgar, que se utiliza, al igual que en la mano humana, como elemento opuesto al resto de dedos. La propagación de las fuerzas ejercidas por el resto de dedos afecta al movimiento del pulgar, porque éste debe contrarrestar las fuerzas de los demás.

Se muestran las gráficas de evolución para las fuerzas y deformaciones en dos de los objetos usados. El primer objeto utilizado es una esponja de tamaño: 0.13 x 0.075 x $0.045 \mathrm{~m}$. Se muestran las evoluciones de los valores de deformación (Figura 5) y las fuerzas ejercidas (Figura 4). El segundo de los objetos usados es una pelota de goma de radio $=0.045 \mathrm{~m}$. Se muestran a las gráficas de evolución de las fuerzas ejercidas (Figura 6) por cada dedo y la deformación (7) causada en cada iteración del algoritmo.

Para el valor de la deformación se usa la notación de deformación usada en ingeniería $e$, teniendo en cuenta una longitud inicial $L$ y la longitud actual $l$ :

$$
e=\frac{l-L}{L}
$$

Dado un valor deseado de deformación $\boldsymbol{\epsilon}_{\boldsymbol{d}}$, se calcula el valor de presión correspondiente $\boldsymbol{\sigma}_{\boldsymbol{d}}$ para provocar 
Para objetos elásticos valores negativos de deformación indican compresión, y valores positivos indican extensión.

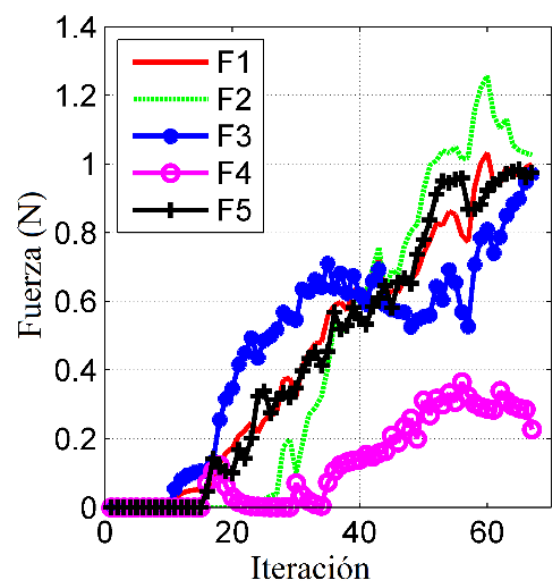

Figura 4: Evolución de las fuerzas aplicadas durante el primer experimento (esponja).

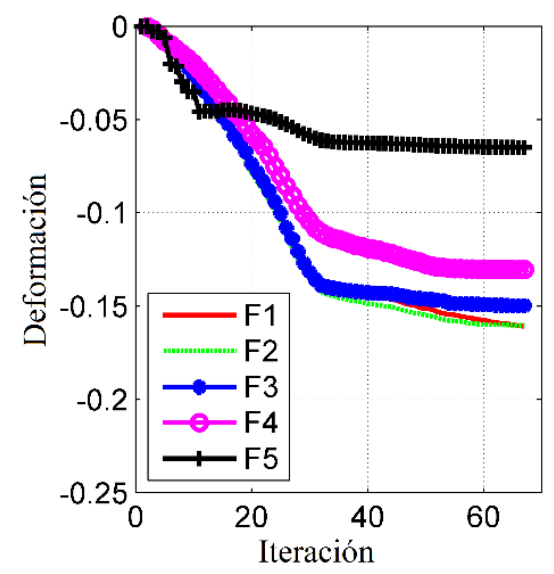

Figura 5: Evolución de la deformación primer experimento (esponja).

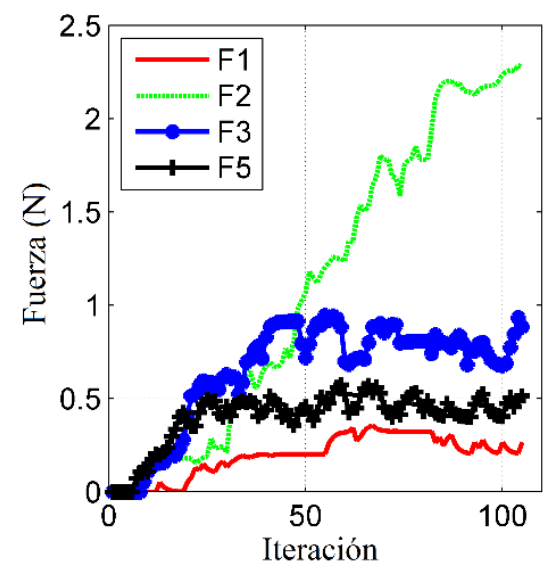

Figura 6: Evolución de las fuerzas aplicadas durante el segundo experimento (pelota).

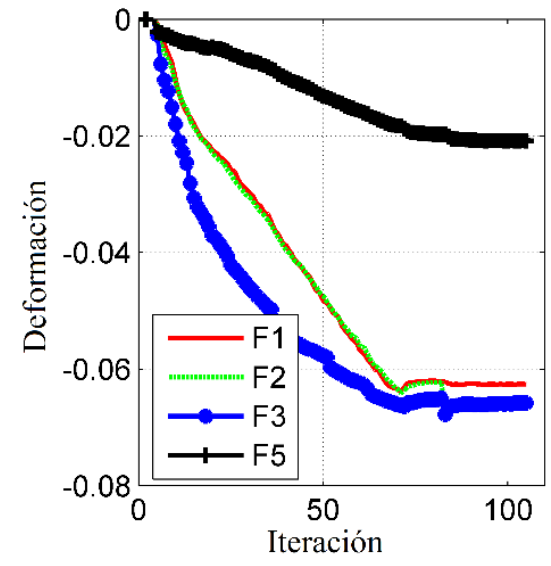

Figure 7: Evolución de la deformación en el segundo experimento (pelota).

En las gráficas se pueden observar las zonas de comportamiento aproximadamente lineal para cada objeto. En el primer objeto se produce en el intervalo entre las iteraciones 1 a 30, a partir de ese punto las fuerzas siguen aumentando por la presión, pero deja de producirse más deformación. En el segundo objeto, entre las iteraciones 1 a 50 se produce un comportamiento lineal, estabilizándose los valores de deformación a partir de ese punto. La siguiente tabla resume los valores estimados para el módulo de Young obtenidos en la etapa de reconocimiento para distintos objetos.

Tabla 1: valores estimados para los distintos objetos deformables:

\begin{tabular}{|c|c|}
\hline Objeto & $\begin{array}{c}\text { Estimación módulo de } \\
\text { Young }\left(\mathrm{N} / \mathrm{cm}^{2}\right)\end{array}$ \\
\hline Esponja & 1.66 \\
\hline Pelota de goma & 2.66 \\
\hline Cilindro semi-rígido & 10.90 \\
\hline $\begin{array}{c}\text { Bloque de corcho } \\
\text { blando }\end{array}$ & 11.28 \\
\hline Espuma de poliuretano & 3.90 \\
\hline
\end{tabular}

\subsection{EXPERIMENTOS PARA EL CONTROL SERVO-TÁCTIL}

La segunda parte de la experimentación consiste en testear la parte del control servo-táctil para los dedos de la mano, que controla la deformación y las fuerzas ejercidas al objeto agarrado durante las tareas de manipulación. El controlador de cada dedo usa como referencia valores de fuerza establecidos según el valor del módulo de Young del objeto usado. Como se ha descrito en las secciones anteriores, si este valor es desconocido, se realiza la etapa de extracción de características. El siguiente ejemplo muestra cómo el control táctil se usa en cada dedo para controlar la deformación causada en uno de los objetos, la pelota de goma. Usando el valor estimado para el módulo de 
Young, se establecen distintas fuerzas objetivo en para los distintos dedos, de manera que se consiga deformar el objeto en los puntos deseados. En la Figura 10 y en la Figura 11 se muestra la evolución de las fuerzas ejercidas y el error respectivamente en un ejemplo en el que se usa un valor de 1 newton como referencia. Se puede observar que las fuerzas convergen a valores muy próximos a la consigna en torno a los 2 segundos de ejecución. La Figura 12 muestra las velocidades de salida obtenidas con el controlador para cada uno de los dedos usados. Los controladores PID se han configurado en este ejemplo con valores $\mathrm{p}=0.5, \mathrm{i}=0.3$, $\mathrm{d}=0.3$.

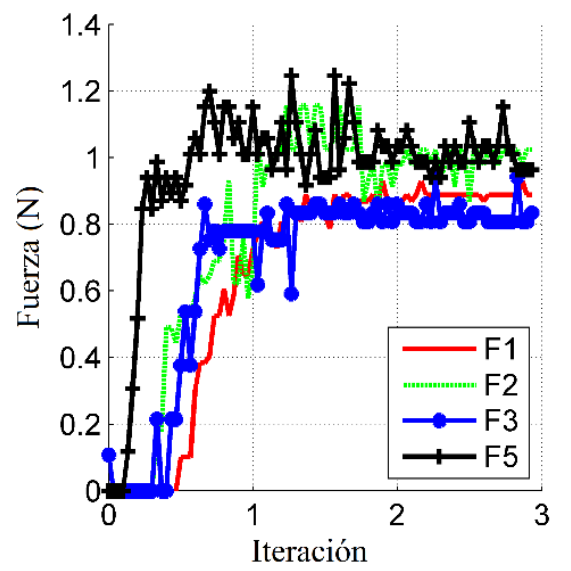

Figura 10: Evolución de las fuerzas aplicadas durante el experimento de control táctil.

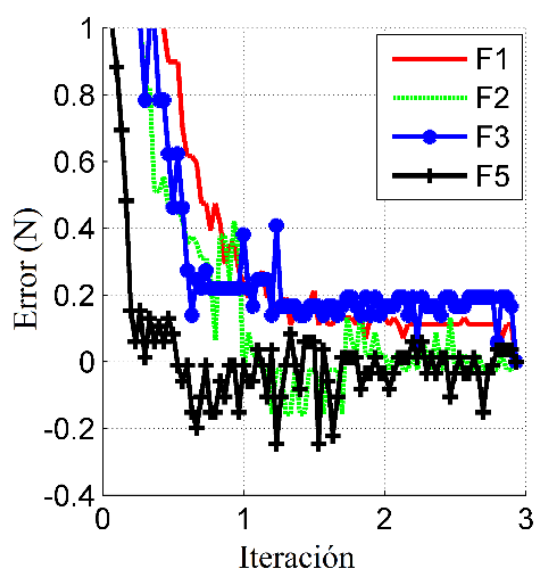

Figura 11: Evolución del error para cada contacto durante el experimento de control táctil.

El principal problema encontrado para aplicar la técnica de control servo-táctil es la aparición de valores inesperados en las medidas de los sensores. Estos errores afectan a la estabilidad del sistema, por lo que se ha recurrido a utilizar un escalado bajo en el error, adaptado a la resolución del sensor, con el fin de minimizar el efecto de altas variaciones en el sensor. A pesar de estas variaciones, se puede observar como las fuerzas convergen y el error es minimizado, controlando de esta manera la deformación causada en el objeto.
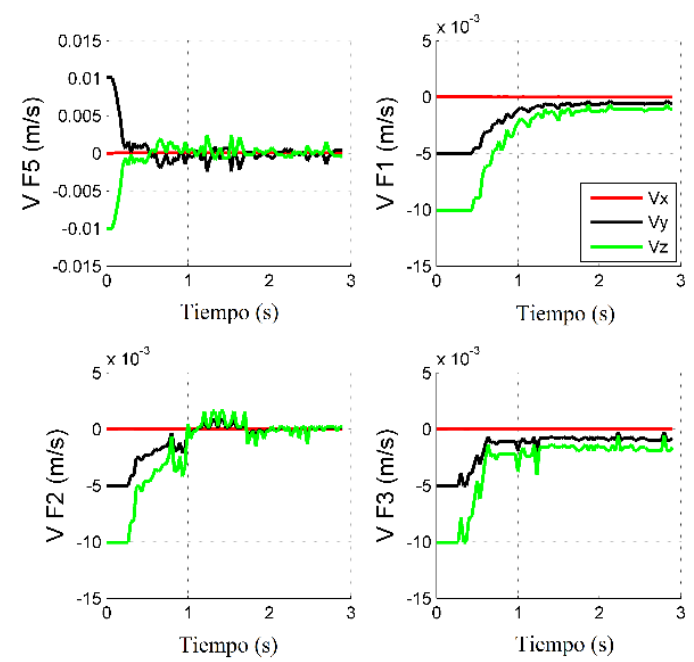

Figura 12: Velocidades de salida de cada controlador.

\section{CONCLUSIONES}

En este artículo se ha presentado una nueva estrategia de control basada en información táctil para tareas de manipulación diestra de objetos deformables. Esta técnica se divide en dos etapas: una etapa de extracción de características y una etapa de control servo-táctil para mantener la presión en los contactos con el objeto con el fin de estabilizar el agarre y controlar el grado de deformación causada en el objeto. Como se ha descrito en los experimentos, la técnica se ha probado con resultados positivos, donde se consigue estabilizar el agarre del objeto con la mano robótica.

La principal contribución de esta técnica es la posibilidad de obtener un comportamiento fiable en tareas de manipulación diestra de objetos deformables, sin necesidad de disponer de un modelo del objeto. Las técnicas que usan modelos del objeto, permiten obtener simulaciones muy precisas de las deformaciones causadas en objetos cuando se ejercen fuerzas sobre ellos. Sin embargo, cuando este control se usa con dispositivos reales, el tiempo de procesamiento necesario para predecir deformaciones y adaptar los movimientos de los dedos, suele ser demasiado alto para hacer tareas en tiempo real. Además, como se ha descrito en el artículo, se puede obtener un comportamiento fiable aun cuando no se dispone de información sobre el comportamiento del objeto, ya que esta información se aproxima de 
manera rápida con el proceso de extracción de características.

Como trabajo futuro, esta técnica se usará como control a nivel bajo en tareas de manipulación. Un controlador a nivel alto, planificará las configuraciones de posición y fuerza en cada momento para llevar a cabo tareas de manipulación diestra automatizadas, en las que se controle la posición y deformación causada en los objetos.

\section{Agradecimientos}

Investigación financiada por el Ministerio de Economía, fondos europeos FEDER y la Generalitat Valenciana, a través de los proyectos DPI2015-68087$\mathrm{R}$ and PROMETEO/2013/085.

\section{Referencias}

[1] Chitta, S., Sturm, J., Piccoli, M., \& Burgard, W. (2011). Tactile sensing for mobile manipulation. IEEE Transactions on Robotics, 27(3), 558-568.

[2] Corrales, J.A., Torres, F., Perdereau, V., 2013. "Finger Readjustment Algorithm for Object Manipulation Based on Tactile Information". Int J Adv Robot Syst, 2013, 10:9. DOI: 10 5772/53561.

[3] Desbrun M., Schroder P., and Barr A., "Interactive animation of structured deformable objects," in Graphics Interface, 1999.

[4] Faure F., Gilles B., Bousquet G., and Pai D. K., "Sparse meshless models of complex deformable solids," SIGGRAPH, vol. 1, no. 212, p. 1, 2011.

[5] Frank B., Stachniss C., Abdo N., and Burgard W., "Efficient motion planning for manipulation robots in environments with deformable objects," in Proc. IEEE/RSJ International Conference on Intelligent Robots and Systems (IROS), Sept. 2011, pp. 21802185.

[6] Kaufmann P., Martin S., Botsch M., and Gross M., "Flexible simulation of deformable models using discontinuous Galerkin FEM," in SIGGRAPH, 2008.

[7] Khalil F. and Payeur P., "Dexterous robotic manipulation of deformable objects with multi-sensory feedback - a review," in Robot Manipulators, Trends and Development, In-Teh, Ed., 2010, ch. 28, pp. 587-621.

[8] Kien-Cuong, N., Perdereau, V., 2013. "Fingertip force control based on max torque adjustment for dexterous manipulation of an anthropomorphic hand". Intelligent Robots and Systems (IROS), 2013 IEEE/RSJ International Conference on vol., no., pp.3557-3563, 37 Nov. 2013.

[9] Li Q., Elbrechter C., Haschke R., Ritter H., "Integrating vision, haptics and proprioception into a feedback controller for in-hand manipulation of unknown objects," in Proc. IEEE/RSJ Int. Conf. on Intelligent Robots and Systems (IROS), Tokyo, 2013, pp. 24662471.

[10] Li, Q., Schürmann, C., Haschke, R., \& Ritter, H. (2013). A control framework for tactile servoing. Proceedings of Robotics: Science and Systems.
[11] Liu, H., Song, X., Nanayakkara, T., Seneviratne, L. D., \& Althoefer, K. (2012). A computationally fast algorithm for local contact shape and pose classification using a tactile array sensor. In Proceedings - IEEE International Conference on Robotics and Automation (pp. 1410-1415).

[12] Luo, Y., \& Nelson, B. J. (2001). Fusing force and vision feedback for manipulating deformable objects. Journal of Robotic Systems, 18(3), 103-117.

[13] Roa M., Suárez R., Cornellà J (2008). Medidas de calidad para la prensión de objetos. Revista iberoamericana de Automatica e Informatica Industrial. 2008 vol: 5 (1) pp: 66-82.

[14] Romano, J. M., Hsiao, K., Niemeyer, G., Chitta, S., \& Kuchenbecker, K. J. (2011). Human-inspired robotic grasp control with tactile sensing. IEEE Transactions on Robotics. http://doi.org/10.1109/TRO.2011.2162271.

[15] Wada T., Hirai S., Kawarnura S., and Karniji N., "Robust manipulation of deformable objects by a simple PID feedback," in Proc. IEEE International Conference on Robotics and Automation (ICRA), 2001.

[16] Wettels, N., \& Loeb, G. E. (2011). Haptic feature extraction from a biomimetic tactile sensor: Force, contact location and curvature. In 2011 IEEE International Conference on Robotics and Biomimetics, ROBIO

2011. http://doi.org/10.1109/ROBIO.2011.6181676.

[17] Yoshikawa T., (2010). Multifingered robot hands: Control for grasping and manipulation. Annual Reviews in Control vol. 34 (2), p. 199-208.

[18] Yousef, H., Boukallel, M., \& Althoefer, K. (2011). Tactile sensing for dexterous in-hand manipulation in robotics-A review. Sensors and Actuators A: Physical, 167(2),

171-187. http://doi.org/10.1016/j.sna.2011.02.038.

[19] Shadow Robot, "Dexterous Hand". [Online]. Available:http://www.shadowrobot.com/products/dexte rous-hand/

[20] Tactile Sensor Tekscan. [Online]. Available: http://www.tekscan.com/grip-pressure-measurement 